\title{
Electron transfer reactions in chemistry. Theory and experiment
}

\author{
R.A. Marcus \\ Noyes Laboratory of Chemical Physics, California Institute of Technology, Pasadena, CA 91125 , \\ USA
}

\section{ELECTRON TRANSFER EXPERIMENTS SINCE THE LATE 1940 S}

Since the late 1940s, the field of electron transfer processes has grown enormously, both in chemistry and biology. The development of the field, experimentally and theoretically, as well as its relation to the study of other kinds of chemical reactions, represents to us an intriguing history, one in which many threads have been brought together. In this lecture, some history, recent trends, and my own involvement in this research are described.

The early experiments in the electron transfer field were on 'isotopic exchange reactions' (self-exchange reactions) and, later, 'cross reactions.' These experiments reflected two principal influences. One of these was the availability after the Second World War of many radioactive isotopes, which permitted the study of a large number of isotopic exchange electron transfer reactions, such as

$\mathrm{Fe}^{2+}+\mathrm{Fe}^{* 3+} \rightarrow \mathrm{Fe}^{3+}+\mathrm{Fe}^{* 2+}$,

and

$\mathrm{Ce}^{3+}+\mathrm{Ce}^{* 4+} \rightarrow \mathrm{Ce}^{4+}+\mathrm{Ce}^{* 3+}$,

in aqueous solution, where the asterisk denotes a radioactive isotope.

There is a two-fold simplicity in typical self-exchange electron transfer reactions (so-called since other methods beside isotopic exchange were later used to study some of them): (1) the reaction products are identical with the reactants, thus eliminating one factor which usually influences the rate of a chemical reaction in a major way, namely the relative thermodynamic stability of the reactants and products; and (2) no chemical bonds are broken or formed in simple electron transfer reactions. Indeed, these self-exchange reactions represent, for these combined reasons, the simplest class of reactions in chemistry. Observations stemming directly from this simplicity were to have major consequences, not only for the electron transfer field but also, to a lesser extent, for the study of other kinds of chemical reactions as well (cf. Shaik et al., ref. 2).

A second factor in the growth of the electron transfer field was the introduction of new instrumentation, which permitted the study of the rates of rapid chemical reactions. Electron transfers are frequently rather fast, compared with many reactions which undergo, instead, a breaking of chemical bonds and a forming of new ones. Accordingly, the study of a large body of fast electron transfer reactions became accessible with the introduction of this instrumentation. One example of the latter was the stopped-flow apparatus, pioneered for inorganic electron transfer reactions by N. Sutin. It permitted the study of bimolecular reactions in solution in the millisecond time scale (a fast time scale at the time). Such studies led to the investigation of what has been termed electron transfer 'cross reactions,' i.e., electron transfer reactions between two different redox systems, as in

$\mathrm{Fe}^{2+}+\mathrm{Ce}^{4+} \rightarrow \mathrm{Fe}^{3+}+\mathrm{Ce}^{3+}$,

which supplemented the earlier studies of the self-exchange electron transfer reactions. A comparative study of these two types of reaction, self-exchange and cross-reactions, stimulated by theory, was also later to have major consequences for the field and, indeed, for other areas.

Again, in the field of electrochemistry, the new post-war instrumentation in chemical laboratories led to methods which permitted the study of fast electron transfer reactions at metal electrodes. Prior to the late 1940 s only relatively slow electrochemical reactions, such as the discharge of an $\mathrm{H}_{3} \mathrm{O}^{+}$ion at an electrode 
to form $\mathrm{H}_{2}$, had been investigated extensively. They involved the breaking of chemical bonds and the forming of new ones.

Numerous electron transfer studies have now also been made in other areas, some depicted in Figure 1. Some of these investigations were made possible by a newer technology, lasers particularly, and now include studies in the picosecond and subpicosecond time regimes. Just recently, (non-laser) nanometersized electrodes have been introduced to study electrochemical processes that are still faster than those hitherto investigated. Still other recent investigations, important for testing aspects of the electron transfer theory at electrodes, involve the new use of an intervening ordered adsorbed monolayer of long chain organic compounds on the electrode to facilitate the study of various effects, such as varying the metalsolution potential difference on the electrochemical electron transfer rate.

In some studies of electron transfer reactions in solution there has also been a skilful blending of these measurements of chemical reaction rates with various organic or inorganic synthetic methods, as well as with site-directed mutagenesis, to obtain still further hitherto unavailable information. The use of chemically modified proteins to study the distance dependence of electron transfer, notably by Gray and co-workers, has opened a whole new field of activity.

The interaction of theory and experiment in these many electron transfer fields has been particularly extensive and exciting, and each has stimulated the other. The present lecture addresses the underlying theory and this interaction.

\section{THE EARLY EXPERIENCE}

My own involvement in the electron transfer field began in a rather circuitous way. In an accompanying biographical note I have commented on my earlier background, which was in experimental measurements of reaction rates as a chemistry graduate student at McGill University (1943-46) and as a post-doctoral associate at the National Research Council of Canada (1946-49). A subsequent post-doctoral study at the University of North Carolina (1949-51) on the theory of reaction rates resulted in what is now known in the literature as RRKM theory (Rice, Ramsperger, Kassel, Marcus).

This unimolecular reaction field reflects another long and extensive interaction between theory and experiment. RRKM theory enjoys widespread use and is now usually referred to in the literature only by its acronym (or by the texts written about it, ref. 4), instead of by citation of the original articles.

After the theoretical post-doctoral I joined the faculty of the Polytechnic Institute of Brooklyn in 1951 and wondered what theoretical research to do next after writing the RRKM papers (1951-52). I remember vividly how a friend of mine, a colleague at Brooklyn Poly, Frank Collins, came down to my office every day with a new idea on the liquid state transport theory which he was developing, while I, for theoretical research, had none. Perhaps this gap in not doing anything immediately in the field of theory was, in

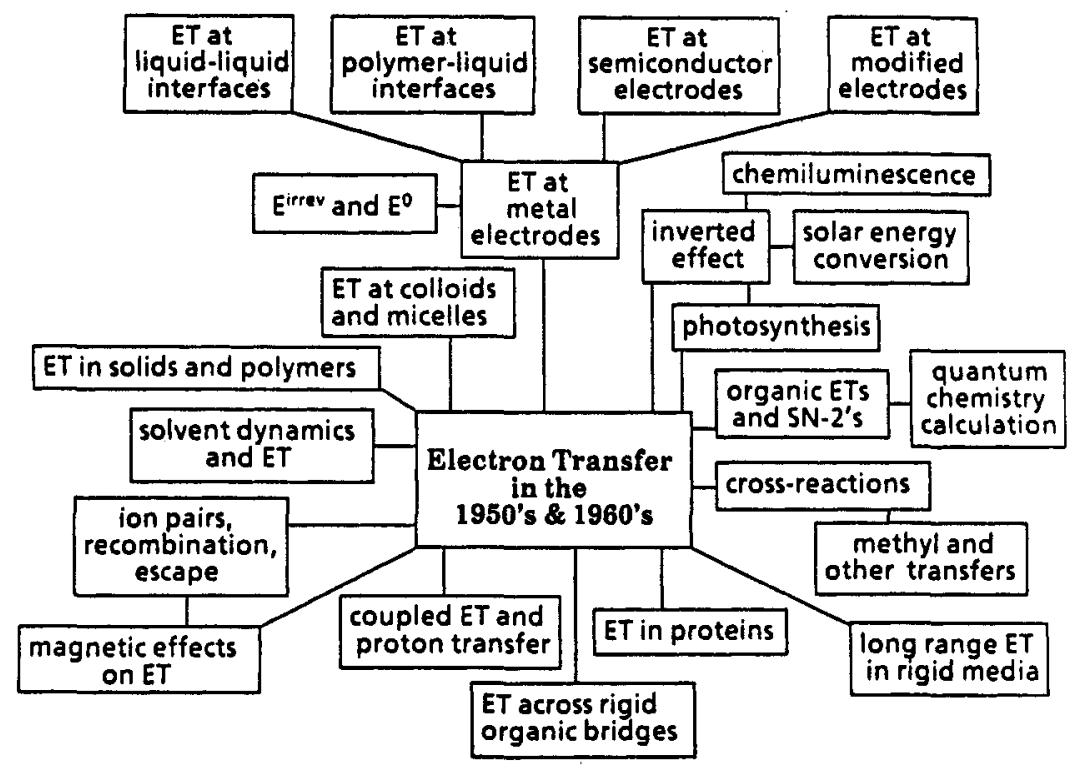

Figure 1. Examples of topics in the electron transfer field (Marcus and Siddarth, ref. 2). 
retrospect, fortunate: In not continuing with the study of the theory of unimolecular reactions, for which there were too few legitimate experimental data at the time to make the subject one of continued interest, I was open for investigating quite different problems in other areas. I did, however, begin a program of experimental studies in gas phase reactions, prompted by my earlier studies at NRC and by the RRKM work.

In the biographical note I have also recalled how a student in my statistical mechanics class in this period (Abe Kotliar) asked me about a particular problem in polyelectrolytes. It led to my writing two papers on the subject (1954-55), one of which required a considerable expansion in my background in electrostatics, so as to analyze different methods for calculating the free energy of these systems: In polyelectrolyte molecules, it may be recalled, the ionic charges along the organic or inorganic molecular backbone interact with each other and with the solvent. In the process I read the relevant parts of the texts that were readily available to me on electrostatics (Caltech's Mason and Weaver's was later to be particularly helpful!). When shortly thereafter I encountered some papers on electron transfer, a field entirely new to me, I was reasonably well prepared for treating the problems which lay ahead.

\section{DEVELOPING AN ELECTRON TRANSFER THEORY}

\section{Introduction}

My first contact with electron transfers came in 1955 as a result of chancing upon a 1952 symposium issue on the subject in the Journal of Physical Chemistry. An article by Bill Libby caught my eye-a use of the Franck-Condon principle to explain some experimental results, namely, why some isotopic exchange reactions which involve electron transfer between pairs of small cations in aqueous solution, such as reaction (1), are relatively slow, whereas electron transfers involving larger ions, such as $\mathrm{Fe}(\mathrm{CN})_{6}{ }^{3-}-$ $\mathrm{Fe}(\mathrm{CN})_{6}{ }^{4}$ and $\mathrm{MnO}_{4}^{-}-\mathrm{MnO}_{4}{ }^{2-}$, are relatively fast.

Libby explained this observation in terms of the Franck-Condon principle, as discussed below. The principle was used extensively in the field of spectroscopy for interpreting spectra for the excitation of the molecular electronic-vibrational quantum states. An application of that principle to chemical reaction rates was novel and caught my attention. In that paper Libby gave a 'back-of-the-envelope' calculation of the resulting solvation energy barrier which slowed the reaction. However, I felt instinctively that even though the idea-that somehow the Franck-Condon principle was involved-seemed strikingly right, the calculation itself was incorrect. The next month of study of the problem was, for me, an especially busy one. To place the topic in some perspective I first digress and describe the type of theory that was used for other types of chemical reaction rates at the time and continues to be useful today.

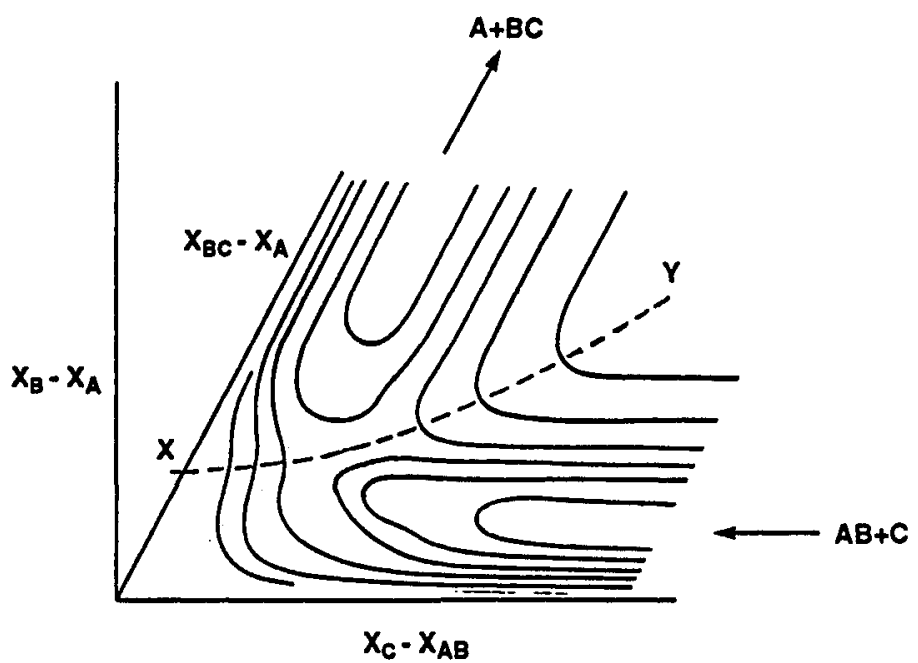

Figure 2 Potential energy contours for reaction (4), $A B+C \rightarrow A+B C$, in the collinear case. 


\section{Reaction rate theory}

Chemical reactions are often described in terms of the motion of the atoms of the reactants on a potential energy surface. This potential energy surface is really the electronic energy of the entire system, plotted versus the positions of all the atoms. A very common example is the transfer of an atom or a group $B$ from $\mathrm{AB}$ to form $\mathrm{BC}$

$\mathrm{AB}+\mathrm{C} \rightarrow \mathrm{A}+\mathrm{BC}$

An example of reaction (4) is the transfer of an $\mathrm{H}$, such as in $\mathrm{IH}+\mathrm{Br} \rightarrow \mathrm{I}+\mathrm{HBr}$, or the transfer of a $\mathrm{CH}_{3}$ group from one aromatic sulfonate to another. To aid in visualizing the motion of the atoms in this reaction, this potential energy function is frequently plotted as constant energy contours in a space whose axes are chosen to be two important relative coordinates such as, in reaction (4), a scaled $A B$ bond length and a scaled distance from the center of mass of $A B$ to $C$, as in Figure 2.

A point representing this reacting system begins its trajectory in the lower right region of the figure in a valley in this plot of contours, the 'valley of the reactants.' When the system has enough energy, appropriately distributed between the various motions, it can cross the 'mountain pass' (saddle-point region) separating the initial valley from the products' valley in the upper left, and so form the reaction products. There is a line in the figure, $\mathrm{XY}$, analogous to the 'continental divide' in the Rocky Mountains in the U.S., which separates systems which could spontaneously flow into the reactants' valley from those which could flow into the products' one. In chemists' terminology this line represents the 'transition state' of the reaction.

In transition state theory a quasi-equilibrium between the transition state and the reactant is frequently postulated, and the reaction rate is then calculated using equilibrium statistical mechanics. A fundamental dynamical basis, which replaces this apparently ad hoc but common assumption of transition state theory and which is perhaps not as well known in the chemical literature as it deserves to be, was given many years ago by the physicist and one-time chemical engineer, Eugene Wigner (1938). He used a classical mechanical description of the reacting system in the many-dimensional space (of coordinates and momenta). Wigner pointed out that the quasi-equilibrium would follow as a dynamical consequence, if each trajectory of a moving point representing the reacting system in this many-dimensional space did not recross the transition state (and if the distribution of the reactants in the reactants' region were a Boltzmann one). In recent times, the examination of this recrossing has been a common one in classical mechanical trajectory studies of chemical reactions. Usually, recrossings are relatively minor, except in nonadiabatic reactions, where they are readily treated (cf discussion, later).

In practice, transition state theory is generalized, so as to include as many coordinates as are needed to describe the reacting system. Further, when the system can 'tunnel' quantum mechanically through the potential energy barrier (the 'pass') separating the two valleys, as for example frequently happens at low energies in H-transfer reactions, the method of treating the passage across the transition state region needs, and has received, refinement. (The principal problem encountered here has been the lack of 'dynamical separability' of the various motions in the transition state region.)

\section{Electron transfer theory. Formulation}

In contrast to the above picture, we have already noted that in simple electron transfer reactions no chemical bonds are broken or formed and so a somewhat different picture of the reaction is needed for the electron transfer reaction.

In his 1952 symposium paper, Libby noted that when an electron is transferred from one reacting ion or molecule to another, the two new molecules or ions formed are in the wrong environment of the solvent molecules, since the nuclei do not have time to move during the rapid electron jump: in reaction (1) a $\mathrm{Fe}^{2+}$ ion would be formed in some configuration of the many nearby dipolar solvent molecules that was appropriate to the original $\mathrm{Fe}^{3+}$ ion. Analogous remarks apply to the newly formed $\mathrm{Fe}^{3+}$ ion in the reaction. On the other hand, in reactions of 'complex ions,' such as those in the $\mathrm{Fe}(\mathrm{CN})_{6}^{-3}-\mathrm{Fe}(\mathrm{CN})_{6}^{-4}$ and $\mathrm{MnO}_{4}^{-}-$ $\mathrm{MnO}_{4}{ }^{2-}$ self-exchange reactions, the two reactants are larger, and so the change of electric field in the vicinity of each ion, upon electron transfer, would be smaller. The original solvent environment would therefore be less foreign to the newly formed charges, and so the energy barrier to reaction would be less.

(C) 1997 IUPAC, Pure and Applied Chemistry 69, 13-29 


\section{Electron Transfer in Solution}

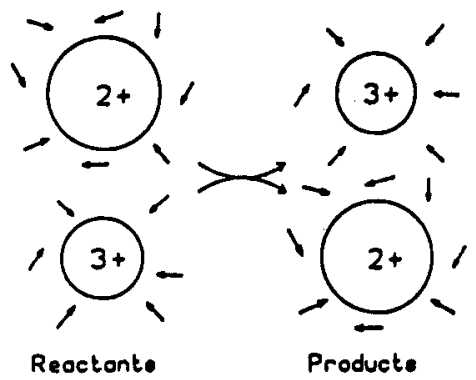

Figure 3. Typical nuclear configurations for reactants, products, and surrounding solvent molecules in reaction (1). The longer $\mathrm{M}-\mathrm{OH}_{2}$ bond length in the +2 state is indicated schematically by the larger ionic radius. (Sutin, ref. 2)

In this way Libby explained the faster self-exchange electron transfer rate for these complex ions. Further confirmation was noted in the ensuing discussion in the symposium: the self-exchange $\mathrm{Co}\left(\mathrm{NH}_{3}\right)_{6}{ }^{3+}-$ $\mathrm{Co}\left(\mathrm{NH}_{3}\right)_{6}{ }^{2+}$ reaction is very slow, and it was pointed out that there was a large difference in the equilibrium $\mathrm{Co}-\mathrm{N}$ bond lengths in the $3+$ and the $2+$ ions, and so each ion would be formed in a very 'foreign' configuration of the vibrational coordinates, even though the ions are 'complex ions.'

After studying Libby's paper and the symposium discussion, I realized that what troubled me in this picture for reactions occurring in the dark was that energy was not conserved: the ions would be formed in the wrong high-energy environment, but the only way such a non-energy conserving event could happen would be by the absorption of light (a 'vertical transition'), and not in the dark. Libby had perceptively introduced the Franck-Condon principle to chemical reactions, but something was missing.

In the present discussion, as well as in Libby's treatment, it was supposed that the electronic interaction of the reactants which causes the electron transfer is relatively weak. That view is still the one that seems appropriate today for most of these reactions. In this case of weak-electronic interaction, the question becomes: how does the reacting system behave in the dark so as to satisfy both the Franck-Condon principle and energy conservation? I realized that fluctuations had to occur in the various nuclear coordinates, such as in the orientation coordinates of the individual solvent molecules and indeed in any other coordinates whose most probable distribution for the products differs from that of the reactants. With such fluctuations, values of the coordinates could be reached which satisfy both the Franck-Condon and energy conservation conditions and so permit the electron transfer to occur in the dark.

For a reaction such as reaction (1), an example of an initial and final configuration of the solvent molecules is depicted in Figure 3. Fluctuations from the original equilibrium ensemble of configurations were ultimately needed, prior to the electron transfer, and were followed by a relaxation to the equilibrium ensemble for the products, after electron transfer.

The theory then proceeded as follows. The potential energy $U_{r}$ of the entire system, reactants plus solvent, is a function of the many hundreds of relevant coordinates of the system, coordinates which include, among others, the position and orientation of the individual solvent molecules (and hence of their dipole moments, for example), and the vibrational coordinates of the reactants, particularly those in any inner coordination shell of the reacting ions. (E.g., the inner coordination shell of an ion such as $\mathrm{Fe}^{2+}$ or $\mathrm{Fe}^{3+}$ in water is known from EXAFS experiments to contain six water molecules.) No longer were there just the two or so important coordinates that were dominant in reaction (4).

Similarly, after the electron transfer, the reacting molecules have the ionic charges appropriate to the reaction products, and so the relevant potential energy function $U_{p}$ is that for the products plus solvent. These two potential energy surfaces will intersect if the electronic coupling which leads to electron transfer is neglected. For a system with $N$ coordinates this intersection occurs on an $(N-1)$ dimensional surface, which then constitutes in our approximation the transition state of the reaction. The neglected electronic coupling causes a well-known splitting of the two surfaces in the vicinity of their intersection. A schematic profile of the two potential energy surfaces in the $N$-dimensional space is given in Figure 4. (The splitting is not shown.) 


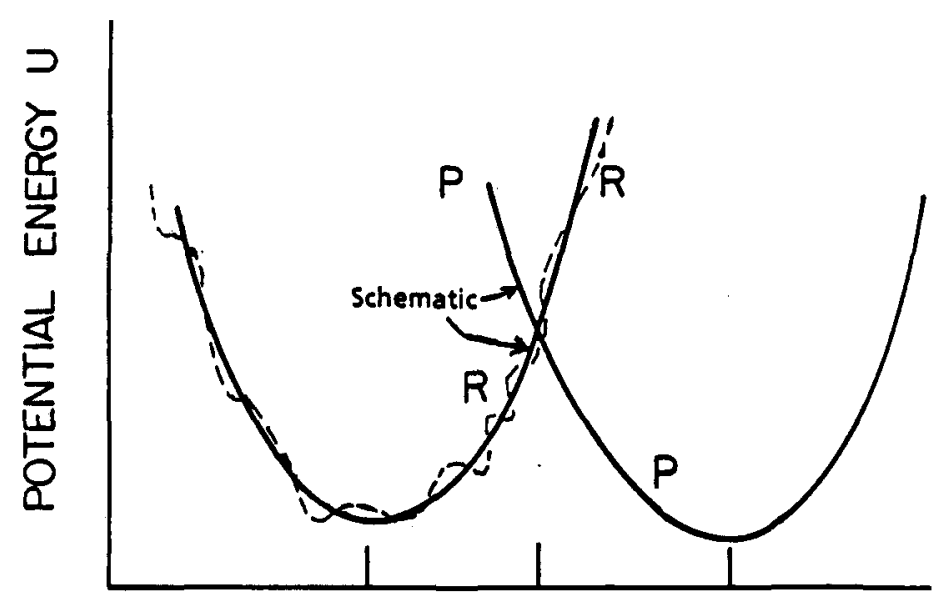

NUCLEAR COORDINATES

Figure 4. Profile of potential energy surfaces for reactants plus environment, $R$, and for products plus environment, $P$. Solid curves: schematic. Dashed curves: schematic but slightly more realistic. The typical splitting at the intersection of $U_{r}$ and $U_{p}$ is not shown in the Figure (Marcus and Siddarth, ref. 2).

Due to the effect of the previously neglected electronic coupling and the coupling to the nudear motion near the intersection surface $S$, an electron transfer can occur at $S$. In classical terms, the transfer at $S$ occurs at fixed positions and momenta of the atoms, and so the Franck-Condon principle is satisfied. Since $U_{r}$ equals $U_{p}$ at $S$, energy is also conserved. The details of the electron transfer depend on the extent of electronic coupling and how rapidly the point representing the system in this $N$-dimensional space crosses $S$. (It has been treated, for example, using as an approximation the well-known one-dimensional LandauZener expression for the transition probability at the near-intersection of two potential energy curves.)

When the splitting caused by the electronic coupling is large enough at the intersection, a system crossing $S$ from the lower surface on the reactants' side of $S$ continues onto the lower surface on the products' side, and so an electron transfer in the dark has then occurred. When the coupling is, instead, very weak, ('nonadiabatic reactions') the probability of successfully reaching the lower surface on the products' side is small and can be calculated using quantum mechanical perturbation theory, for example, using Fermi's 'Golden Rule,' an improvement over the 1-dimensional Landau-Zener treatment.

Thus, there is some difference and some similarity with a more conventional type of reaction such as reaction (4), whose potential energy contour plots were depicted in Figure 2. In both cases, fluctuations of coordinates are needed to reach the transition state, but since so many coordinates can now play a significant role in the electron transfer reaction, because of the major and relatively abrupt change in charge distribution on passing through the transition state region, a rather different approach from the conventional one was needed to formulate the details of the theory.

\section{Electron transfer theory. Treatment}

In the initial paper (1956) I formulated the above picture of the mechanism of electron transfer and, to make the calculation of the reaction rate tractable, treated the solvent as a dielectric continuum. 'In the transition state the position-dependent dielectric polarization $\mathbf{P}_{u}(\mathbf{r})$ of the solvent, due to the orientation and vibrations of the solvent molecules, was not the one in equilibrium with the reactants' or the products' ionic charges. It represented instead, some macroscopic fluctuation from them. The electronic polarization for the solvent molecules, on the other hand, can rapidly respond to any such fluctuations and so is that which is dictated by the reactants' charges and by the instantaneous $\mathbf{P}_{u}(\mathbf{r})$.

With these ideas as a basis, what was then needed was a method of calculating the electrostatic free energy $G$ of this system with its still unknown polarization function $\mathbf{P}_{\mathcal{U}}(\mathbf{r})$. I obtained this free energy $G$ by finding a reversible path for reaching this state of the system. Upon then minimizing $G$, subject to the constraint imposed by the Franck-Condon principle (reflected in the electron transfer occurring at the 
intersection of the two potential energy surfaces), I was able to find the unknown $\mathbf{P}_{u}(\mathbf{r})$ and, hence, to find the $G$ for the transition state. That $G$ was then introduced into transition state theory and the reaction rate calculated.

In this research I also read and was influenced by a lovely paper by Platzmann and Franck (1952) on the optical absorption spectra of halide ions in water and later by work of physicists such as Pekar and Frohlich (1954) on the closely related topic of polaron theory. As best as I can recall now, my first expressions for $G$ during this month of intense activity seemed rather clumsy, but then with some rearrangement a simple expression emerged that had the right 'feel' to it and that I was also able to obtain by a somewhat independent argument. The expression also reduced reassuringly to the usual one, when the constraint of arbitrary $\mathbf{P}_{u}(\mathbf{r})$ was removed. Obtaining the result for the mechanism and rate of electron transfer was indeed one of the most thrilling moments of my scientific life.

The expression for the rate constant $k$ of the reaction is given by

$k=A \exp \left(\frac{-\Delta G^{*}}{k_{B} T}\right)$,

where $\Delta G^{*}$, in turn, is given by

$\Delta G^{*}=\frac{\lambda}{4}\left(1+\frac{\Delta G^{0}}{\lambda}\right)^{2}$

The $A$ in Eq. (5a) is a term depending on the nature of the electron transfer reaction (e.g., bimolecular or intramolecular), $\Delta G^{0}$ is the standard free energy of reaction (and equals zero for a self-exchange reaction), $\lambda$ is a 'reorganization term,' composed of solvational $\left(\lambda_{0}\right)$ and vibrational $\left(\lambda_{1}\right)$ components.

$\lambda=\lambda_{0}+\lambda_{i}$

In a two-sphere model of the reactants, $\lambda_{0}$ was expressed in terms of the two ionic radii $a_{1}$ and $a_{2}$ (including in the radius any inner coordination shell), the center-to-center separation distance $R$ of the reactants, the optical $\left(D_{o p}\right)$ and static $\left(D_{S}\right)$ dielectric constants of the solvent, and the charge transferred $\Delta e$ from one reactant to the other:

$\lambda_{0}=(\Delta e)^{2}\left(\frac{1}{2 a_{1}}+\frac{1}{2 a_{2}}-\frac{1}{R}\right)\left(\frac{1}{D_{o p}}-\frac{1}{D_{s}}\right)$

For a bimolecular reaction, work terms, principally electrostatic, are involved in bringing the reactants together and in separating the reaction products, but are omitted from Eq. (5) for notational brevity. The expression for the vibrational term $\lambda_{l}$ is given by

$\lambda_{i}=\frac{1}{2} \sum_{j} k_{j}\left(Q_{j}^{r}-Q_{j}^{p}\right)^{2}$

where $Q_{j}^{r}$ and $Q_{j}^{p}$ are equilibrium values for the $j$ th normal mode coordinate $Q, k_{j}$ is a reduced force constant $2 k_{j}^{r} k_{j} p /\left(k_{j}^{r}+k_{j}^{p}\right)$ associated with it, $k_{j}^{r}$ being the force constant for the reactants and $k_{j}^{p}$ being that for the products. (I introduced a 'symmetrization' approximation for the vibrational part of the potential energy surface, to obtain this simple form of Eqs. (5) to (8), and tested it numerically.)

In 1957 I published the results of a calculation of the $\lambda \mathrm{i}$ arising from a stretching vibration in the innermost coordination shell of each reactant, (the equation used for $\lambda i$ was given in the 1960 paper). An early paper on the purely vibrational contribution using chemical bond length coordinates and neglecting bond-bond correlation had already been published for self-exchange reactions by George and Griffiths in 1956. 
I also extended the theory to treat electron transfers at electrodes, and distributed it as an Office of Naval Research Report in 1957, the equations being published later in a journal paper in 1959. I had little prior knowledge of the subject, and my work on electrochemical electron transfers was facilitated considerably by reading a beautiful and logically written survey article of Roger Parsons on the equilibrium electrostatic properties of electrified metal-solution interfaces.

In the 1957 and 1965 work I showed that the electrochemical rate constant was again given by Eqs. (5)(7), but with $A$ now having a value appropriate to the different 'geometry' of the encounter of the participants in the reaction. The $1 / 2 a_{2}$ in Eq. (7) was now absent (there is only one reacting ion) and $R$ now denotes twice the distance from the center of the reactant's charge to the electrode (it equals the ion-image distance). A term $e \eta$ replaced the $\Delta G^{0}$ in Eq. (5b), where $e$ is the charge transferred between the ion and the electrode, and $\eta$ is the activation overpotential, namely the metal-solution potential difference, relative to the value it would have if the rate constants for the forward and reverse reactions were equal. These rate constants are equal when the minima of the two $G$ curves in Figure 5 have the same height.

When $|e \eta|<\lambda$, most electrons go into or out of quantum states in the metal that are near the Fermi level. However, because of the continuum of states in the metal, the inverted effect was now predicted to be absent for this process, i.e., the counterpart of Eq. (5) is applicable only in the region $|e \eta|\langle\lambda$ : In the case of an intrinsically highly exothermic electron transfer reaction at an electrode, the electron can remove the immediate 'exothermicity' by (if entering) going into a high unoccupied quantum state of the metal, or (if leaving) departing from a low occupied quaritum state, each far removed from the Fermi level. (The inverted region effect should, however, occur for the electron transfer when the electrode is a narrow band semiconductor.)

After these initial electron transfer studies, which were based on a dielectric continuum approximation for the solvent outside the first coordination shell of each reactant, I introduced a purely molecular treatment of the reacting system. Using statistical mechanics, the solvent was treated as a collection of dipoles in the 1960 paper, and later in 1965 a general charge distribution was used for the solvent molecules and for the reactants. At the same time I found a way in this 1960 paper of introducing rigorously a global reaction coordinate in this many-dimensional $(N)$ coordinate space of the reacting system. Thi globally defined coordinate so introduced was equivalent to using $U_{p}-U_{r}$, the potential energy difference between the products plus solvent $\left(U_{p}\right)$ and the reactants plus solvent $\left(U_{r}\right)$ (cf A. Warshel, 1987). It was, thereby, a coordinate defined everywhere in this $N$-dimensional space.

The free energy $G_{r}$ of a system containing the solvent and the reactants, and that of the corresponding system for the products, $G_{p}$, could now be defined along this globally defined reaction coordinate. (In contrast, in reactions such as that depicted by Figure 2 , it is customary, instead, to define a reaction coordinate locally, namely, in the vicinity of a path leading from the valley of the reactants through the saddle point region and into the valley of the products.)

The potential energies $U_{r}$ and $U_{p}$ in the many-dimensional coordinate space are simple functions of the vibrational coordinates but are complicated functions of the hundreds of relevant solvent coordinates: there are many local minima corresponding to locally stable arrangements of the solvent molecules. However, I introduced a 'linear response approximation,' in which any hypothetical change in charge of the reactants produces a proportional change in the dielectric polarization of the solvent. (Recently, I utilized a central limit theorem to understand this approximation better-beyond simple perturbation theory, and plan to submit the results for publication shortly.) With this linear approximation the free energies $G_{r}$ and $G_{p}$ became simple quadratic functions of the reaction coordinate.

Such an approach had major consequences. This picture permitted a depiction of the reaction in terms of parabolic free energy plots in simple and readily visualized terms, as in Figure 5. With them the trends predicted from the equations were readily understood. It was also important to use the free energy curves, instead of oversimplified potential energy profiles, because of the large entropy changes which occur in many electron transfer cross-reactions, due to changes in strong ion-polar solvent interactions, (The free energy plot is legitimately a one-coordinate plot while the potential energy plot is at most a profile of the complicated $U_{r}$ and $U_{p}$ in $N$-dimensional space.

With the new statistical mechanical treatment of 1960 and 1965 one could also see how certain relations between rate constants initially derivable from the dielectric continuum-based equations in the 1956 paper could also be valid more generally. The relations were based, in part, on Equations (4), (5) and (initially via 
(7) and (8)) on the approximate relation

$\lambda_{12} \cong 1 / 2\left(\lambda_{11}+\lambda_{12}\right)$,

where $\lambda_{12}$ is the $\lambda$ for the cross-reaction and the $\lambda_{11}$ and $\lambda_{22}$ are those of the self-exchange reactions.

\section{Predictions}

In the 1960 paper I had listed a number of theoretical predictions resulting from these equations, in part to stimulate discussion with experimentalists in the field at a Faraday Society meeting on oxidation-reduction reactions, where this paper was to be presented. At the time I certainly did not anticipate the subsequent involvement of the many experimentalists in testing these predictions. Among the latter was one which became one of the most widely tested aspects of the theory, namely, the 'cross-relation.' This expression, which follows from Eqs. (5) and (9), relates the rate constant $k_{12}$ of a cross-reaction to the two selfexchange rate constants, $k_{11}$ and $k_{22}$, and to the equilibrium constant $k_{12}$ of the reaction.

$k_{12} \cong\left(k_{11} k_{22} K_{12} f_{12}\right)^{1 / 2}$,

where $f_{12}$ is a known function of $k_{11}, k_{22}$ and $K_{12}$ and is usually close to unity.

Another prediction in the 1960 paper concerned what I termed there the inverted region: In a series of

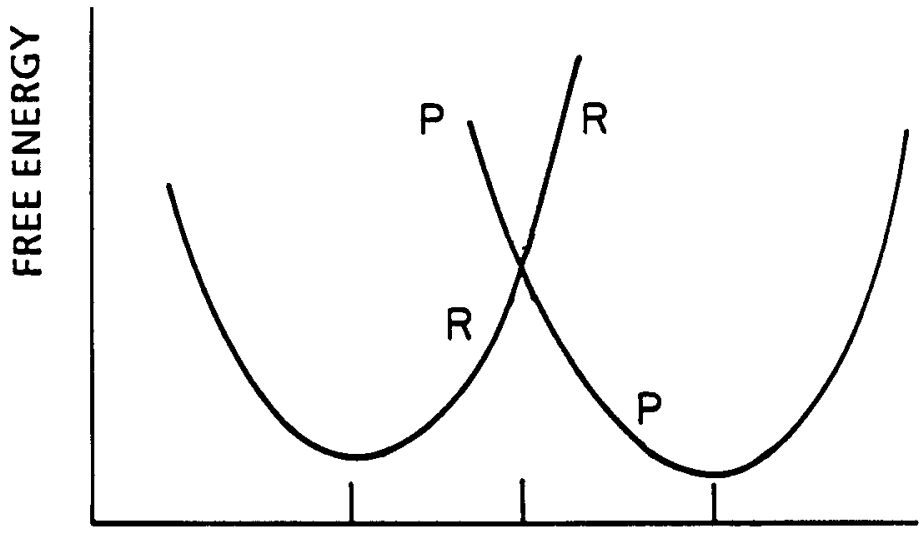

REACTION COORDINATE q

Figure 5. Free energy of reactants plus environment vs. the reaction coordinate $q$ ( $R$ curve), and free energy of products plus environment vs. reaction coordinate $q$ ( $P$ curve). The three vertical lines on the abscissa denote, from left to right, the value for the reactants, for the transition state, and for the products. (Marcus and Siddarth, ref. 2).

related reactions, similar in $\lambda$ but differing in $\Delta G^{0}$, a plot of the activation free energy $\Delta G^{*}$ vs. $\Delta G^{0}$ is seen from Eq. (5) to first decrease as $\Delta G^{0}$ is varied from 0 to some negative value, vanish at $\Delta G^{0}=-\lambda$, and then increase when $\Delta G^{0}$ is made still more negative. This initial decrease of $\Delta G^{*}$ with increasingly negative $\Delta G^{0}$ is the expected trend in chemical reactions and is similar to the usual trend in 'Bronsted plots' of acid or base catalyzed reactions and in 'Tafel plots' of electrochemical reactions. I termed that region of $\Delta G^{0}$ the 'normal' region. However, the prediction for the region where $-\Delta G^{0}>\lambda$, the 'inverted region,' was the unexpected behavior, or at least unexpected until the present theory was introduced.

This inverted region is also easily visualized using Figures 6 and 7: Successively making $\Delta G^{0}$ more negative, by lowering the products' $G$ curve vertically relative to the reactant curve, decreases the free energy barrier $\Delta G^{*}$ (given by the intersection of the reactants' and products' curves): that barrier is seen in Figure 6 to vanish at some $\Delta G^{0}$ and then to increase again.

Other predictions dealt with the relation between the electrochemical and the corresponding selfexchange electron transfer rates, the numerical estimate of the reaction rate constant $k$ and, in the case of non-specific solvent effects, the dependence of the reaction rate on solvent dielectric properties. The testing of some of the predictions was delayed by an extended sabbatical in 1960-61, which I spent auditing courses and attending seminars at the nearby Courant Mathematical Institute. 


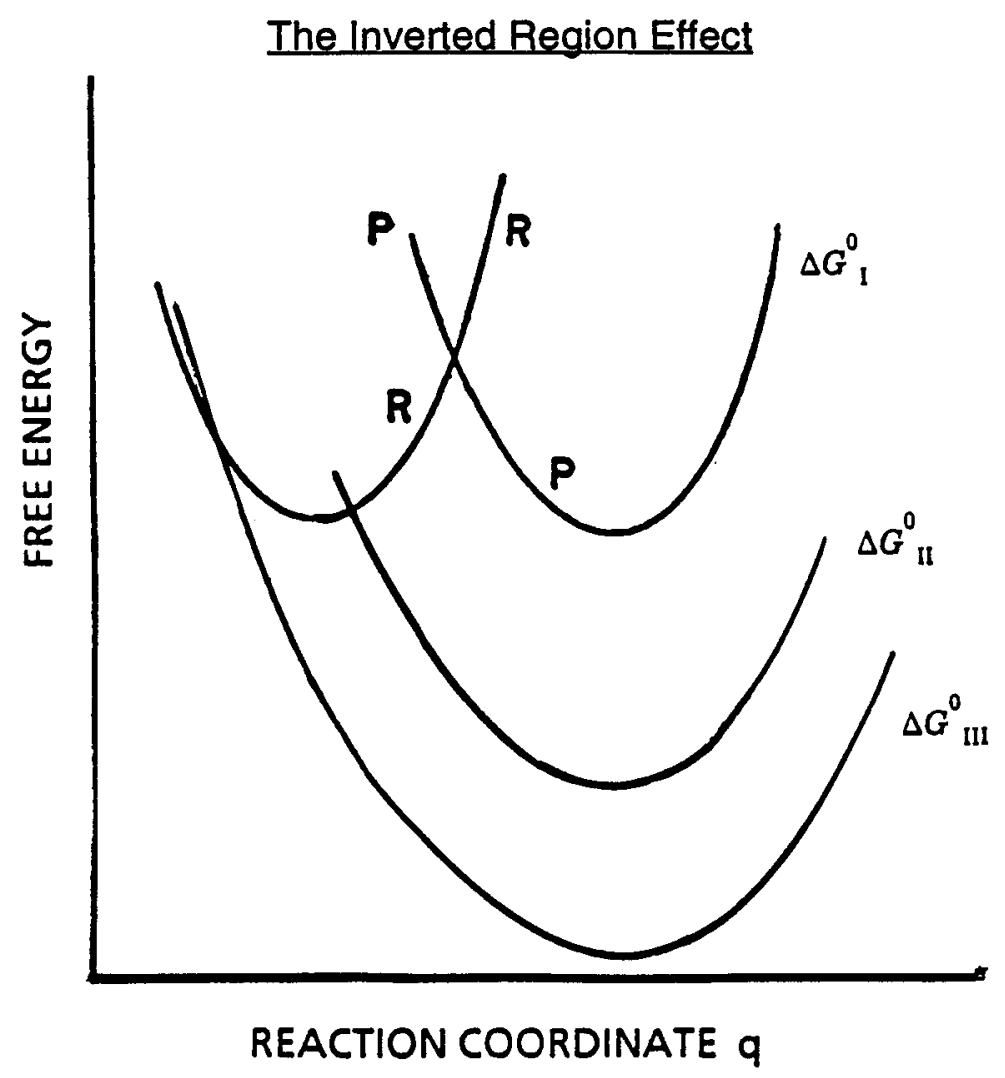

Figure 6. Plot of the free energy $G$ versus the reaction coordinate $q$, for reactants' $(R)$ and products' $(P)$, for three different values of $\Delta G^{0}$, the cases I to $I I I$ indicated in Figure 7 (Marcus and Siddarth, ref. 2).

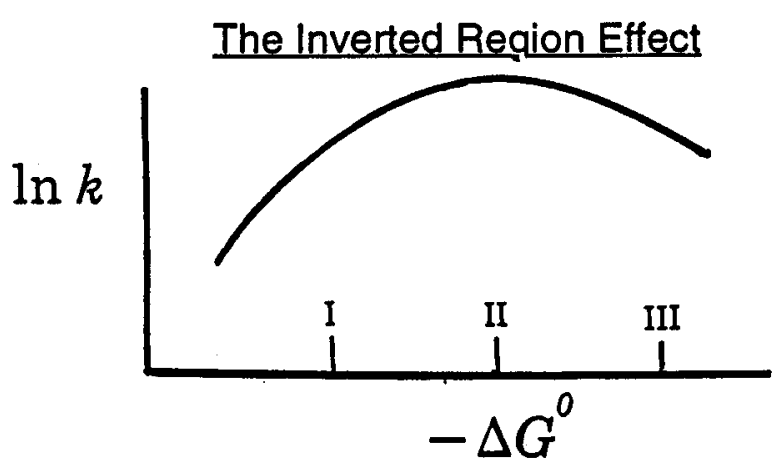

Figure 7. Plot of $\ln k_{r}$ vs $-\Delta G^{0}$. Points I and III are in the normal and inverted regions, respectively, while point II, where $\ln k_{r}$ is a maximum, occurs at $-\Delta G^{0}=\lambda$ (Marcus and Siddarth, ref. 2).

\section{Comparisons of Experiment and Theory}

Around 1962 during one of my visits to Brookhaven National Laboratory, I showed Norman Sutin the 1960 predictions. Norman had either measured via his stopped-flow apparatus or otherwise knew rate constants and equilibrium constants which permitted the cross-relation Eq. (10) to be tested. There were about six such sets of data which he had available. I remember vividly the growing sense of excitement we both felt as, one by one, the observed $k_{12}$ 's more or less agreed with the predictions of the relation. I later collected the results of this and of various other tests of the 1960 predictions and published them in 1963. Perhaps by showing that the previously published expressions were not mere abstract formulae, but rather 
had concrete applications, this 1963 paper, and many tests by Sutin and others, appear to have stimulated numerous subsequent tests of the cross-relation and of the other predictions. A few examples of the crossrelation test are given in Table $I$.

TABLE I. Comparison of calculated and experimental $k_{12}$ values."

\begin{tabular}{|c|c|c|}
\hline \multirow[b]{2}{*}{ Reaction } & \multicolumn{2}{|c|}{$k_{12}\left(M^{-1} \sec ^{-1}\right)$} \\
\hline & Observed & Calculated \\
\hline $\mathrm{IrCl}_{6}{ }^{2-}+\mathrm{W}(\mathrm{CN})_{8}{ }^{4-}$ & $6.1 \times 10^{7}$ & $6.1 \times 10^{7}$ \\
\hline $\mathrm{IrCl}_{6}^{2-}+\mathrm{Fe}(\mathrm{CN})_{6}^{4-}$ & $3.8 \times 10^{5}$ & $7 \times 10^{5}$ \\
\hline $\mathrm{IrCl}_{6}{ }^{2-}+\mathrm{Mo}(\mathrm{CN})_{8}^{4-}$ & $1.9 \times 10^{6}$ & $9 \times 10^{5}$ \\
\hline $\mathrm{Mo}(\mathrm{CN})_{8}{ }^{3-}+\mathrm{W}(\mathrm{CN})_{8}{ }^{4-}$ & $5.0 \times 10^{6}$ & $4.8 \times 10^{6}$ \\
\hline $\mathrm{Mo}(\mathrm{CN})_{8}^{3-}+\mathrm{Fe}(\mathrm{CN})_{6}^{4-}$ & $3.0 \times 10^{4}$ & $2.9 \times 10^{4}$ \\
\hline $\mathrm{Fe}(\mathrm{CN})_{6}{ }^{3-}+\mathrm{W}(\mathrm{CN})_{8}^{4-}$ & $4.3 \times 10^{4}$ & $6.3 \times 10^{4}$ \\
\hline $\mathrm{Ce}^{\mathrm{IV}}+\mathrm{W}(\mathrm{CN})_{8}^{4-}$ & $>10^{8}$ & $4 \times 10^{8}$ \\
\hline $\mathrm{Ce}^{\mathrm{IV}}+\mathrm{Fe}(\mathrm{CN})_{6}^{4-}$ & $1.9 \times 10^{6}$ & $8 \times 10^{6}$ \\
\hline $\mathrm{Ce}^{\mathrm{IV}}+\mathrm{Mo}(\mathrm{CN})_{6}^{4-}$ & $1.4 \times 10^{7}$ & $1.3 \times 10^{7}$ \\
\hline L-Co $[(-) \text { PDTA }]^{2-}+\mathrm{Fe}(\text { bipy })_{3}{ }^{3+}$ & $8.1 \times 10^{4}$ & $\geq 10^{5}$ \\
\hline L-Fe $[(-) \text { PDTA }]^{2-}+\mathrm{Co}(\mathrm{EDTA})^{-}$ & $1.3 \times 10^{1}$ & $1.3 \times 10^{1}$ \\
\hline L-Fe $[(-) \text { PDTA }]^{2-}+\operatorname{Co}(o x)_{3}{ }^{3-}$ & $2.2 \times 10^{2}$ & $1.0 \times 10^{3}$ \\
\hline $\mathrm{Cr}(\mathrm{EDTA})^{2-}+\mathrm{Fe}(\mathrm{EDTA})^{-}$ & $\geq 10^{6}$ & $10^{9}$ \\
\hline $\mathrm{Cr}(\text { EDTA })^{2-}+\mathrm{Co}(\mathrm{EDTA})^{-}$ & $\simeq 3 \times 10^{5}$ & $4 \times 10^{7}$ \\
\hline $\mathrm{Fe}(\mathrm{EDTA})^{2-}+\mathrm{Mn}(\mathrm{CyDTA})^{-}$ & $\simeq 4 \times 10^{5}$ & $6 \times 10^{6}$ \\
\hline $\mathrm{Co}(\mathrm{EDTA})^{2-}+\mathrm{Mn}(\mathrm{CyDTA})$ & $9 \times 10^{-1}$ & 2.1 \\
\hline $\mathrm{Fe}(\text { PDTA })^{2-}+\mathrm{Co}(\mathrm{CyDTA})^{-}$ & $1.2 \times 10^{1}$ & $1.8 \times 10^{1}$ \\
\hline $\mathrm{Co}(\text { terpy })_{2}{ }^{2+}+\mathrm{Co}(\text { bipy })_{3}{ }^{3+}$ & $6.4 \times 10$ & $3.2 \times 10$ \\
\hline $\mathrm{Co}(\text { terpy })_{2}{ }^{2+}+\mathrm{Co}(\text { phen })_{3}{ }^{3+}$ & $2.8 \times 10^{2}$ & $1.1 \times 10^{2}$ \\
\hline $\mathrm{Co}(\text { terpy })_{2}{ }^{2+}+\mathrm{Co}($ bipy $)\left(\mathrm{H}_{2} \mathrm{O}\right)_{4}{ }^{3+}$ & $6.8 \times 10^{2}$ & $6.4 \times 10^{4}$ \\
\hline $\mathrm{Co}(\text { terpy })_{2}{ }^{2+}+\mathrm{Co}($ phen $)\left(\mathrm{H}_{2} \mathrm{O}\right)_{4}{ }^{3+}$ & $1.4 \times 10^{3}$ & $6.4 \times 10^{4}$ \\
\hline $\mathrm{Co}(\text { terpy })_{2}^{2+}+\mathrm{Co}\left(\mathrm{H}_{2} \mathrm{O}\right)_{6}^{3+}$ & $7.4 \times 10^{4}$ & $2 \times 10^{10}$ \\
\hline $\mathrm{Fe}(\text { phen })_{3}^{2+}+\mathrm{MnO}_{4}^{-}$ & $6 \times 10^{3}$ & $4 \times 10^{3}$ \\
\hline $\mathrm{Fe}(\mathrm{CN})_{6}^{4-}+\mathrm{MnO}_{4}^{-}$ & $1.3 \times 10^{4}$ & $5 \times 10^{3}$ \\
\hline $\mathrm{V}\left(\mathrm{H}_{2} \mathrm{O}\right)_{6}{ }^{2+}+\mathrm{Ru}\left(\mathrm{NH}_{3}\right)_{6}{ }^{3+}$ & $1.5 \times 10^{3}$ & $4.2 \times 10^{3}$ \\
\hline $\mathrm{Ru}(\mathrm{en})_{3}{ }^{2+}+\mathrm{Fe}\left(\mathrm{H}_{2} \mathrm{O}\right)_{6}{ }^{3+}$ & $8.4 \times 10^{4}$ & $4.2 \times 10^{5}$ \\
\hline $\mathrm{Ru}\left(\mathrm{NH}_{3}\right)_{6}{ }^{2+}+\mathrm{Fe}\left(\mathrm{H}_{2} \mathrm{O}\right)_{6}{ }^{3+}$ & $3.4 \times 10^{5}$ & $7.5 \times 10^{6}$ \\
\hline $\mathrm{Fe}\left(\mathrm{H}_{2} \mathrm{O}\right)_{6}{ }^{2+}+\mathrm{Mn}\left(\mathrm{H}_{2} \mathrm{O}\right)_{6}{ }^{3+}$ & $1.5 \times 10^{4}$ & $3 \times 10^{4}$ \\
\hline
\end{tabular}

'Bennett, Ref. 3.

The encouraging success of the experimental tests given in the 1963 paper suggested that the theory itself was more general than the approximations (e.g., solvent dipoles, unchanged force constants) used in 1960 and stimulated me to give a more general formulation (1965). The latter paper also contains a unified treatment of electron transfers in solution and at metal electrodes, and served, thereby, to generalize my earlier (1957) treatment of the electrochemical electron transfers.

The best experimental evidence for the inverted region was provided in 1984 by Miller, Calcaterra and Closs, almost 25 years after it was predicted. This successful experimental test, which was later obtained for other electron transfer reactions in other laboratories, is reproduced in Figure 8. Possible reasons for not observing it in the earlier tests are several-fold and have been discussed elsewhere.

Previously, indirect evidence for the inverted region had been obtained by observing that electron transfer reactions with a very negative $\Delta G^{*}$ may result in chemiluminescence: when the $G_{r}$ and $G_{p}$ curves intersect at a high $\Delta G^{*}$ because of the inverted region effect, there may be an electron transfer to a more easily accessible $G_{p}$ curve, one in which one of the products is electronically excited and which intersects the $G_{r}$ curve in the normal region at a low $\Delta G^{*}$, as in Figure 9. Indeed, experimentally in some reactions $100 \%$ formation of an electronically excited state of a reaction product has been observed by Bard and coworkers, and results in chemiluminescence.

Another consequence of Eq. (5) is the linear dependence of $k_{B} T \ln k$ on $-\Delta G^{0}$ with a slope of $1 / 2$, when | $\Delta G^{0} / \lambda \mid$ is small, and a similar behavior at electrodes, with $\Delta G^{0}$ replaced by $e \eta$, the product of the charge 


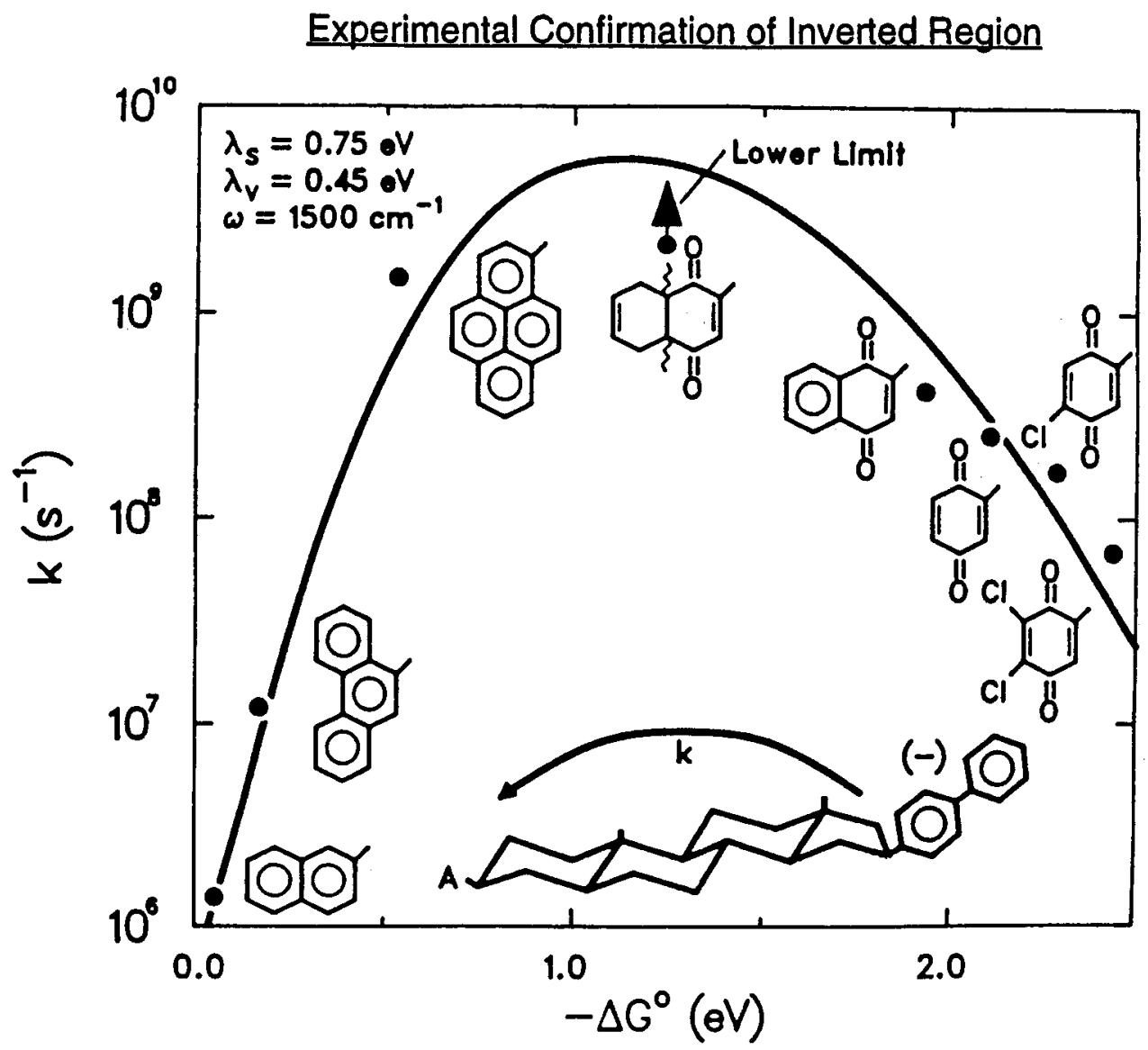

Figure 8. Inverted region effect in chemical electron transfer reactions. (Miller, et al, ref. 3).

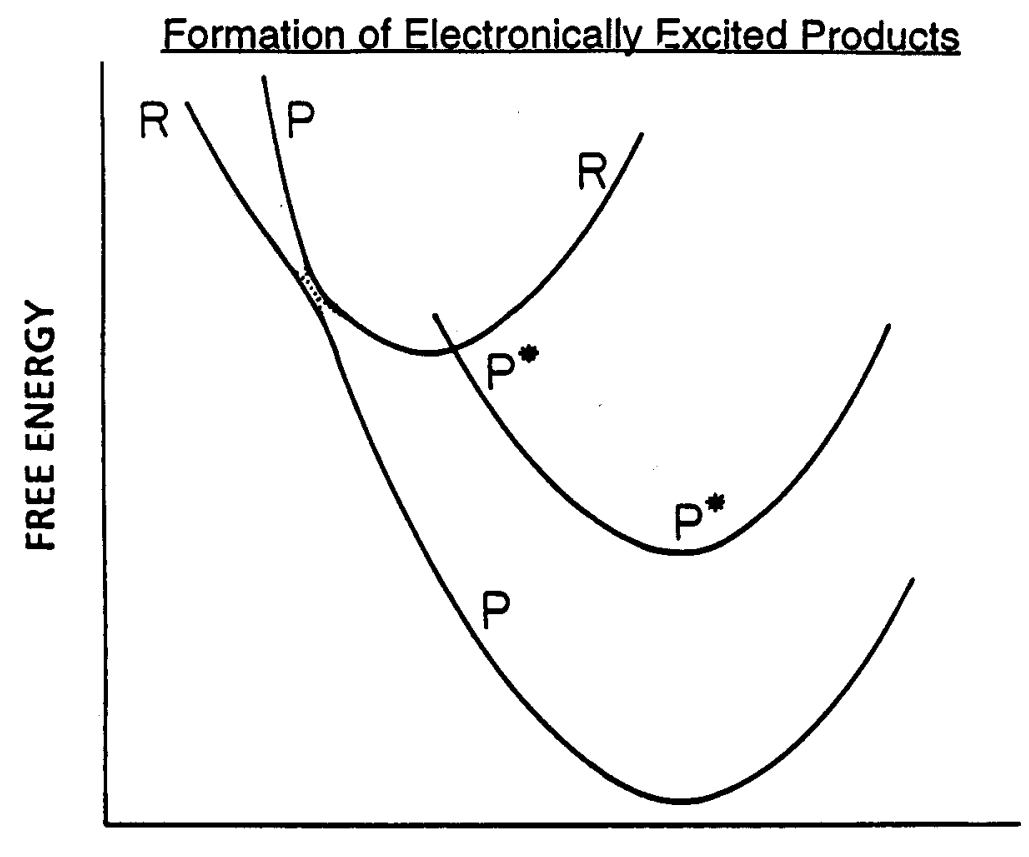

REACTION COORDINATE q

Figure 9. A favored formation of an electronically excited state of the products (Marcus and Siddarth, ref. 2). 
transferred and the activation overpotential. Extensive verification of both these results has been obtained. More recently, the curvature of plots of $\ln k$ vs. e $\eta$, expected from these equations, has been demonstrated in several experiments. The very recent use of ordered organic molecular monolayers on electrodes, either to slow down the electron transfer rate or to bind a redox-active agent to the electrode, but in either case to avoid or minimize diffusion control of the fast electron transfer processes, has considerably facilitated this study of the curvature in the $\ln k v s$. en plot.

Comparison of experiment and theory has also included that of the absolute reaction rates of the selfexchange reactions, the effect on the rate of varying the solvent, an effect sometimes complicated by ion pairing in the low dielectric constant media involved, and studies of the related problem of charge transfer spectra, such as

$\mathrm{DA}+h v \rightarrow \mathrm{D}^{+} \mathrm{A}^{-}$

Here, the frequency of the spectral absorption maximum $v_{\max }$ is given by

$h v_{\max }=\lambda+\Delta G^{0}$

Comparisons with Eq. (12), using Eq. (7) for $\lambda$, have included those of the effects of separation distance and of the solvent dielectric constant.

Comparisons have also been made of the self-exchange reaction rates in solution with the rates of the corresponding electron transfer reactions at electrodes. An example of the latter is the plot given in Figure 10 , where the self-exchange rates are seen to vary by some twenty orders of magnitude. The discrepancy at high $k$ 's is currently the subject of some reinvestigation of the fast electrode reaction rates, using the new nanotechnology. Most recently, a new type of interfacial electron transfer rate has also been measured, electron transfer at liquid-liquid interfaces. In treating the latter, I extended the 'cross relation' to this twophase system. It is clear that much is to be learned from this new area of investigation. (The study of the transfer of ions across such an interface, on the other hand, goes back to the time of Nernst and of Planck, around the turn of the century.)

\section{Electrochemical vs Self-Exchange Rate Constants}

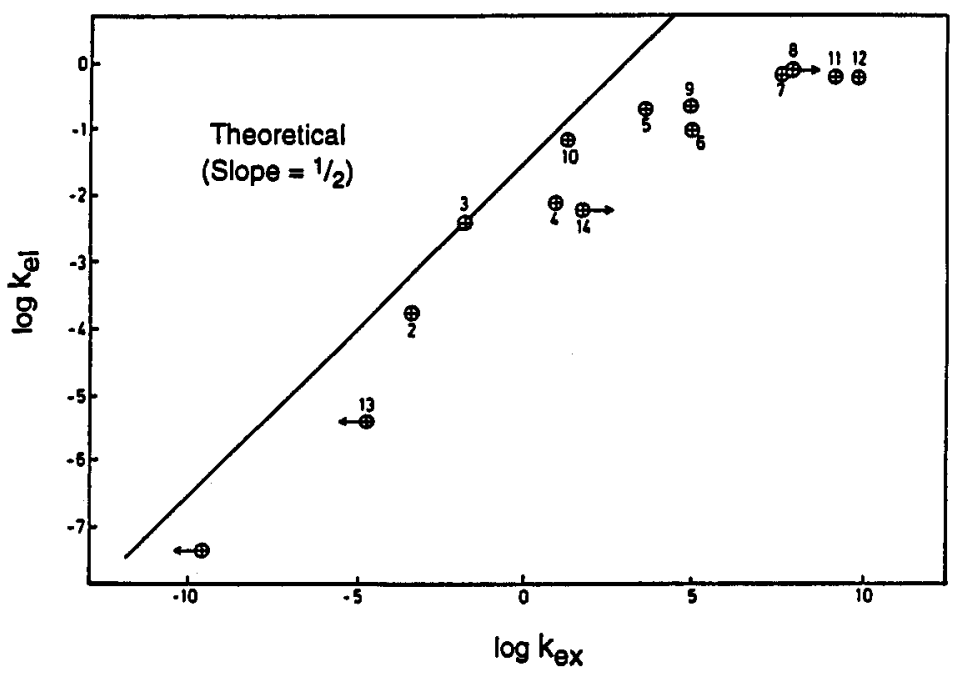

Figure 10. Comparison of isotopic exchange electron transfer rates in solution, covering 20 orders of magnitude, with rates of corresponding electron transfers at metal electrodes. (Cannon, ref. 2)

\section{Other Applications and Extensions}

As noted in Figure 1, one aspect of the electron transfer field has been its continued and, indeed, everexpanding growth in so many directions. One of these is in the biological field, where there are now detailed experimental and theoretical studies in photosynthetic and other protein systems. The three- 


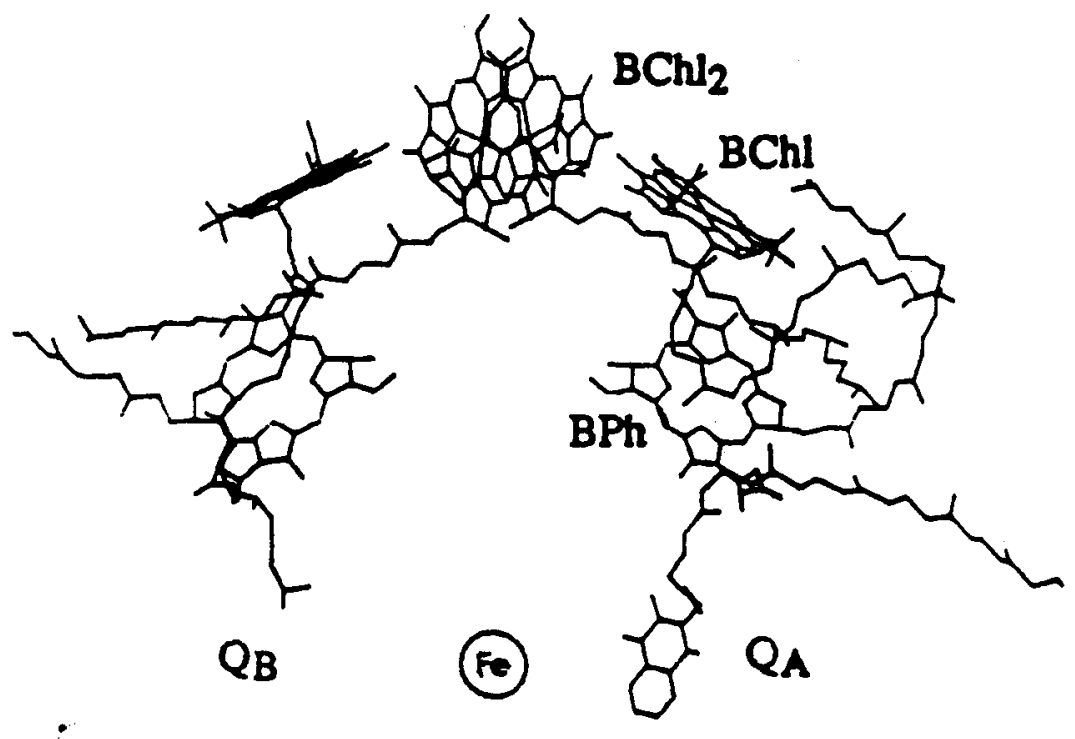

Figure 11. Redox-active species involved in the initial charge separation for a photosynthetic bacterium (Deisenhofer et al, ref. 3; cf Yeates et al, ref. 3), with labels added, to conform to the present text; they include a missing $Q_{B}$.

dimensional structure of a photosynthetic reaction center, the first membrane protein to be so characterized, was obtained by Deisenhofer, Michel and Huber, who received the Nobel Prize in Chemistry in 1988 for this work. A bacterial photosynthetic system is depicted in Figure 11, where the protein framework holding fast the constituents in this reaction center is not shown.

In the photosynthetic system there is a transfer of electronic excitation from 'antenna' chlorophylls (not shown in Figure 11) to a special pair $\mathrm{BChl}_{2}$. The latter then transfers an electron to a pheophytin $\mathrm{BPh}$ within a very short time ( $\sim 3$ picoseconds) and from it to a quinone $Q_{A}$ in $200 \mathrm{psec}$ and thence to the other quinone $Q_{B}$. (Other chemical reactions then occur with these separated charges at each side of the membrane, bridged by this photosynthetic reaction center.)

To avoid wasting the excitation energy of the $\mathrm{BChl}_{2}{ }^{*}$ unduly it is necessary that the $-\Delta G^{0}$ of this first electron transfer to $\mathrm{BPh}$ be small. (It is only about $0.25 \mathrm{eV}$ out of an overall excitation energy of $\mathrm{BChl}_{2}{ }^{*}$ of $1.38 \mathrm{eV}$.) In order that this electron transfer also be successful in competing with two wasteful processes, the fluorescence and the radiationless transition of $\mathrm{BChl}_{2}{ }^{*}$, it is also necessary that $\Delta G^{*}$ for that first electron transfer step be small and hence, by Eq. (5), that the $\lambda$ be small.

The size of the reactants is large, and the immediate protein environment is largely nonpolar, so leading to a small $\lambda$ (cf Eq.(7)). Nature appears, indeed, to have constructed a system with this desirable property.

Furthermore, to avoid another form of wasting the energy, it is also important that an unwanted back electron transfer reaction from the $\mathrm{BPh}^{-}$to the $\mathrm{BChl}_{2}^{+}$not compete successfully with a second forward electron transfer step from $\mathrm{BPh}^{-}$to $\mathrm{Q}_{\mathrm{A}}$. That is, it is necessary that the back transfer, a "hole-electron recombination' step, be slow, even though it is a very highly exothermic process $(\sim 1.1 \mathrm{eV})$. It has been suggested that the small $\lambda(\sim 0.25 \mathrm{eV})$ and the resulting inverted region effect play a significant role in providing this essential condition for the effectiveness of the photosynthetic reaction center.

There is now a widespread interest in synthesizing systems which can mimic the behavior of nature's photosynthetic systems, and so offer other routes for the harnessing of solar energy. The current understanding of how nature works has served to provide some guidelines. In this context, as well as that of electron transfer in other proteins, there are also relevant experiments in long range electron transfer. Originally the studies were of electron transfer in rigid glasses and were due to Miller and coworkers. More recently the studies have involved a donor and receptor held together by synthetically made rigid molecular bridges. The effect of varying the bridge length has been studied in the various systems. A theoretical estimate of the distance dependence of electron transfers in a photosynthetic system was first made by Hopfield, who used a square barrier model and an approximate molecular estimate of the barrier height. 
Recently, in their studies of long range electron transfer in chemically modified proteins, Gray and coworkers have studied systematically the distance or site dependence of the electronic factor, by attaching an appropriate electron donor or acceptor to a desired site. For each such site the reactant chosen should be such that $-\Delta G^{0} \approx \lambda$, i.e., which has a $k$ at the maximum of the $\ln k$ vs. $-\Delta G^{0}$ curve (cf Eqs. (4)-(5)). The value of $k$ then no longer depends on a $\Delta G^{*}$. Since $\Delta G^{*}$ is distance-dependent (cf Eq.(7)), it is particularly desirable to make $\Delta G^{*} \approx 0$, so that the relative $k^{\prime}$ 's at the various sites now reflect only the electronic factor. Dutton and coworkers have treated data similarly for a number of reactions by using, where possible, the $k$ at the maximum of each $\ln k v s$. $\Delta G^{0}$ curve. Of particular interest in such studies is whether there is a simple exponential decrease of the electronic factor on the separation distance between donor and acceptor, or whether there are deviations from this monotonic behavior, due to local structural factors.

In a different development, the mechanism of various organic reactions has been explored by several investigators, notably by Eberson (ref. 2), in the light of current electron transfer theory. Other organic reactions have been explored by Shaik and Pross, in their analysis of a possible electron transfer mechanism vs. a conventional mechanism, and by Shaik et al. (ref. 2).

Theoretical calculations of the donor-acceptor electronic interactions, initially by McConnell and by Larsson, and later by others, our group among them, have been used to treat long-range electron transfer. The methods have recently been adapted to large protein systems. In our studies with Siddarth we used an 'artificial intelligence' searching technique to limit the number of amino acids used in the latter type of study.

Another area of much current activity in electron transfers is that of solvent dynamics, following the pioneering treatment for general reactions by Kramers (1940). Important later developments for electron transfer were made by many contributors. Solvent dynamics affects the electron transfer reaction rate when the solvent is st ' ficiently sluggish. As we showed recently with Sumi and Nadler, the solvent dynamics effect can also be modified significantly, when there are vibrational $\left(\lambda_{i}\right)$ contribution to $\lambda$.

Computational studies, such as the insightful one of David Chandler and coworkers on the $\mathrm{Fe}^{2+}+\mathrm{Fe}^{3+}$ self-exchange reaction, have also been employed recently. Using computer simulations they obtained a verification of the parabolic $G$ curves, even for surprisingly high values of the fluctuation in $G$. They also extended their studies to dynamical and quantum mechanical effects of the nuclear motion. Studies of the quantum mechanical effects on the nuclear motion on electron transfer reactions were initiated in 1959 by Levich and Dogonadze, who assumed a harmonic oscillator model for the polar solvent medium and employed perturbation theory. Their method was related to that used for other problems by Huang and Rhys (1951) and Kubo and Toyozawa (1954).

There were important subsequent developments by various authors on these quantum effects, including the first discussion of quantum effects for the vibrations of the reactants by Sutin in 1962 and the important work of Jortner and coworkers in 1974-75, who combined a Levich and Dogonadze type approach to treat the high frequency vibrations of the reactants with the classical expression which I described earlier for the polar medium. These quantum effects have implications for the temperature dependence of $k$, among other effects. Proceeding in a different (classical) direction Savéant recently showed how to extend Eq. (5) to reactions which involved the rupture of a chemical bond by electron transfer and which he had previously studied experimentally: $M(e)+R X \rightarrow M+R+X^{-}$, where $R$ is an alkyl group, $X$ a halide and $M$ a metal electrode.

A particularly important early development was that by Taube in the 1950 s, who received the Nobel Prize for his work in 1983. Taube introduced the idea of different mechanisms for electron transfer-outer sphere and inner sphere electron transfers, which he had investigated experimentally. His experimental work on charge transfer spectra of strongly interacting systems ('Creutz-Taube' ion, 1959, 1973) and of weakly interacting ones has been similarly influential. Also notable has been Hush's theoretical work on charge transfer spectra, both of intensities and absorption maxima (1967), which supplemented his earlier theoretical study of electron transfer rates (1961).

There has been a 'spin-off' of the original electron transfer theory to other types of chemical reactions as well. In particular, the $\Delta G^{*}$ vs $\Delta G^{0}$ relation and the cross-relation have been extended to these other reactions, such as the transfer of atoms, protons, or methyl groups. (Even an analog of Eqs. (5) and (9), but for binding energies instead of energy barriers has been introduced to relate the stability of isolated protonbound dimers $\mathrm{AHB}^{+}$to those of $\mathrm{AHA}^{+}$and $\mathrm{BHB}^{+}$!) 
Since the transfer of these nuclei involves strong electronic interactions, it is not well represented by intersecting parabolic free energy curves, and so a different theoretical approach was needed. For this purpose I adapted (1968) a 'bond-energy-bond-order' model of H. Johnston, in order to treat the problem for a reaction of the type given by Eq. (4). The resulting simple expression for $\Delta G^{*}$ is similar to Eq. (5), when $\left|\Delta G^{0} / \lambda\right|$ is not large $(<1 / 2)$, but differs from it in not having any inverted region. It has the same $x$ property as that given by Eq. (9), and has resulted in a cross-relation analogous to Eq. (10). The crossrelation has been tested experimentally for the transfer of methyl groups by E. Lewis, and the $\Delta G^{*}$ vs. $\Delta G^{0}$ relation has been used or tested for other transfers by Albery and by Kreevoy and their coworkers, among others.

It is naturally gratifying to see one's theories used. A recent article, which showed the considerable growth in the use of papers such as the 1956 and 1964 articles (ref. 5), points up the impressive and continued vitality of the field itself. The remarks above on many areas of electron transfer and on the spinoff of such work on the study of other types of reactions represent a necessarily brief picture of these broad-based investigations.

\section{ACKNOWLEDGEMENTS}

My acknowledgements are to my many fellow researchers in the electron transfer field, notably Norman Sutin, with whom I have discussed so many of these matters for the past thirty or more years. I also thank my students and post-doctorals, whose presence was a constant source of stimulation to me, both in the electron transfer field and in the other fields of research which we have explored. In its earliest stage and for much of this period this research was supported by the Office of Naval Research and also later by the National Science Foundation. The support of both agencies continues to this day and I am very pleased to acknowledge its value and timeliness here.

In my Nobel lecture, I concluded on a personal note with a slide of my great-uncle, Henrik Steen (né Markus), who came to Sweden in 1892 . He received his doctorate in theology from the University of Uppsala in 1915, and was an educator and a prolific writer of pedagogic books. As I noted in the biographical sketch in Les Prix Nobel, he was one of my childhood idols. Coming here, visiting with my Swedish relatives-some thirty or so of his descendants-has been an especially heartwarming experience for me and for my family. In a sense I feel that I owed him a debt, and that it is most fitting to acknowledge that debt here.

\section{REFERENCES}

Some of my relevant articles, largely from the 1956-65 period, are listed in ref. 1 below, and some general references which review the overall literature are listed in ref. 2. Several additional references for the Table and for the Figures are given in ref. 3. Classic texts on unimolecular reactions are given in ref. 4.

1. R. A. Marcus, J. Chem. Phys. 24, 966 (1956); ibid., 24, 979 (1956); ibid., 26, 867 (1957); ibid., 26, 872 (1957); Trans. N. Y. Acad. Sci. 19, 423 (1957); ONR Technical Report No. 12, Project NR 051-331 (1957), reproduced in Special Topics in Electrochemistry, P. A. Rock, ed., Elsevier, New York, 1977, p. 181; Can. J. Chem. 37, 155 (1959); Discussions Paraday Soc. 29, 21 (1960); J. Phys. Chem. 67, 853, 2889 (1963); J. Chem. Phys. 38, 1858 (1963); ibid., 39, 1734 (1963); Ann. Rev. Phys. Chem. 15, 155 (1964); J. Chem. Phys. 43, 679 (1965); ibid., 43, 1261 (1965); ibid., 43, 2654 (1965), (corr.) 52, 2803 (1970); J. Phys. Chenı. 72, 891 (1968)

2. R.A. Marcus and N. Sutin, Biochim. Biophys. Acta, 811, 265 (1985); J.R. Bolton, N. Mataga and G. McLendon (eds.) Adv. Chem. Ser., 228, (1991), assorted articles; M.D. Newton and N. Sutin, Ann. Rev. Phys. Chem., 35, 437 (1984); N. Sutin, Prog. Inorg. Chem., 30, 441 (1983); M.D. Newton, Chem. Rev., 91, 767 (1991); R.D. Cannon, Electron Transfer Reactions, Butterworths, London, 1980; L. Eberson, Electron Transfer Reactions in Organic Chemistry, Springer, New York, 1987; M.A. Fox and M. Chanon (eds.), Photoinduced Electron Transfer, Elsevier, New York, 1988, 4 vols.; M.V. Twigg (ed.), Mechanisms of Inorganic and Organometallic Reactions, vol. 7, 1991, Chaps. 1 and 2, and earlier volumes; R. A. Marcus and P. Siddarth, in Photoprocesses in Transition Metal Complexes, Biosystems and Other Molesules: Experiment and Theory, E. Kochanski, ed., Kluwer, Norwall, Massachusetts, 1992, p. 49; S.S. Shaik, H.B. Schlegel and S. Wolfe, Theoretical Aspects of Physical Organic Chemistry, J. Wiley, New York, 1992; N. Sutin, Pure \& Applied Chem. 60, 1817 (1988); Assorted articles in Chem. Revs. 92, No. 3 (1992); R.A. Marcus Commemorative Issue, J. Phys. Chem. 90, 
(1986). An important pair of articles on quantum effects, inadvertently omitted in my Les Prix Nobel article, by Ovchinnikov and Ovchinnikova (1969), and the monograph by J. Ulstrup (1979) should also have been mentioned.

3. L.E. Bennett, Prog. Inorg.Chem. 18, 1 ( 1973); J.R. Miller, L.T. Calcaterra and G.L. Closs, J. Am. Chem. Soc. 106, 3047 (1984); J. Deisenhofer, O. Epp, K. Miki, R. Huber and H. Michel, J. Mol. Biol. 180, 385 (1984); J. Deisenhofer and H. Michel, Angew. Chem. Int. Ed. Engl. 28, 829 (1989); T.G. Yeates, H. Komiya, D.C. Rees, J.P. Allen and G. Feher, Proc. Nat. Acad. Sci. 84, 6438 (1987).

4. P.J. Robinson and H.A. Holbrook, Unimolesular Reactions, J.Wiley, New York 1972; W. Forst, Theory of Unimolesular Reactions, Academic Press, New York, 1973; cf also the very recent text, R.G. Gilbert and S.C. Smith, Theory of Unimolesular and Recombination Reactions, Blackwells, Oxford, 1990.

5. Science Watch, 3, No. 9, November, 1992, p. 8. 